\title{
EXACT SOLUTION OF THE HARMONIC PROBLEM FOR A RECTANGULAR PLATE IN FLAT DEFORMATION BY THE METHOD OF INITIAL FUNCTIONS
}

\author{
E.T. OLODO* \\ Polytechnic School of Abomey-Calavi (EPAC) \\ Department of Civil Engineering, University of Abomey-Calavi \\ 01BP2009 Cotonou, BENIN \\ E-mail: olodoe@live.fr \\ V. ADANHOUNME and M.N. HOUNKONNOU \\ International Chair in Mathematical Physics and Applications \\ (ICMPA-UNESCO Chair), University of Abomey-Calavi (UAC) \\ 072 BP 50 Cotonou, BENIN \\ E-mail: adanhounmvillvo@yahoo.fr \\ norbert.hounkonnou@cipma.uac.bj
}

\begin{abstract}
A wide range of applications is based nowadays on analytical developments which allow a precise and effective approach and short time of computations compared with the time required for numerical methods; in this way these developments are suitable for calculations in real time. This work proposes an approach for solving a two-dimensional harmonic problem of a rectangular plate under local surface loading using Vlasov's symbolic method of initial functions and a general solution of the harmonic equation for a rectangle. Substituting the harmonic functions in symbolic form for the corresponding solutions allows us to give the exact solution of the problem in trigonometric form.
\end{abstract}

Key words: harmonic functions, initial functions, flat deformation.

\section{Introduction}

Many problems in solid mechanics are described by mathematical models. In many cases the development of these models requires solving partial differential equations. Analytical methods for solving these equations are the main tools for understanding these problems. However, during the past decades the important growth of computing capabilities allowed numerical solving of complex problems both geometrical point of view and the laws of behavior of materials. This ability to model complex mechanical systems by numerical methods contributed to reduce the interest to analytical solutions. New analytical methods have been developed for solving the boundary problems in elasticity [1-4]. Among these methods one can cite Vlasov's method of initial functions (MIF) which is often used for solving the boundary problems. This method is a powerful one for obtaining an exact solution for some types of problems in the theory of elasticity without hypotheses about the character of the strain-stress state of the structural element. MIF was first proposed by Maliev [5] and further developed by Vlasov [6]. The authors attempted to find a rational method for obtaining harmonic and biharmonic functions for the general analysis in the theory of elasticity, i.e., represent the displacements and stresses via some functions. On the basis of this rational

\footnotetext{
* To whom correspondence should be addressed
} 
method, they constructed the functions of general analysis with the initial functions by virtue of special differential operators proposed by Kellog [7].

MIF has been used intensively for the analysis of various engineering problems. For example, threedimensional elasticity equations for circular cylindrical shells are solved by assuming Taylor series expansions for the stresses and displacements by Faraji et al. [8]; an elasto-plastic problem of a circular plate subjected to antisymmetric lateral load can be found in [9]; a circular cylindrical shell under antisymmetric load is studied in [10]; an analysis of composite beams and other flexural members using MIF can be found in $[11,12]$; an algorithm for constructing the basic equations of MIF and the convergence of power series is studied by Matrosov [13].

In this work, we proposed an approach for solving the two-dimensional harmonic problem of a rectangular plate under local surface loading, using the symbolic method of initial functions and the general solution of the harmonic equation for a rectangle.

\section{Materials and method}

The principle of the method is to determine a vector $\mathrm{W}$ representing the components of strain-stress state of an elastic system in the Cartesian coordinate system such as

$$
W=\{w \sigma\} .
$$

Here, $w=\{u(x, y, z) ; v(x, y, z) ; \omega(x, y, z)\}$ is the displacement vector;

$\sigma=\left\{\sigma_{z}(x, y, z) ; \tau_{y z}(x, y, z) ; \tau_{x z}(x, y, z) ; \sigma_{x}(x, y, z) ; \sigma_{y}(x, y, z) ; \tau_{x y}(x, y, z)\right\}$ the stress vector.

Then the equilibrium equations will be written in the following form

$$
L W=0
$$

where $L$ is the differential operator.

The general solution to this three dimensional problem of elasticity theory is given by

$$
\begin{aligned}
& U=L_{U U} U_{0}+L_{U V} V_{0}+\ldots+L_{U X} \tau_{X Z_{0}}, \\
& V=L_{V U} U_{0}+L_{V V} V_{0}+\ldots+L_{V X} \tau_{X Z_{0}}, \\
& X=L_{X U} U_{0}+L_{X V} V_{0}+\ldots+L_{X X} \tau_{X Z_{0}}
\end{aligned}
$$

where, $L_{U U}, L_{U V}, \ldots, L_{X X}$ are the differential operators related to the initial functions

$U_{0}, V_{0}, \tau_{x z_{0}}$ depending on $x$ and $y$ on the plane $Z=0$. Each type of problems has its own operatorfunctions $L_{i j}$, and consists differential operators, geometrical parameters and elastic constants.

So, in symbolic form for a band and using Vlasov's general symbolic solution [14] the solution of the problem in plane strain can be expressed as

$$
\begin{aligned}
& U(x, y)=G u(x, y)=L_{U U} U_{0}(x)+L_{U V} V_{0}(x)+L_{U Y} Y_{0}(x)+L_{U X} X_{0}(x), \\
& V(x, y)=G v(x, y)=L_{V U} U_{0}(x)+L_{V V} V_{0}(x)+L_{V Y} Y_{0}(x)+L_{V X} X_{0}(x),
\end{aligned}
$$




$$
\begin{aligned}
& Y(x, y)=\sigma_{y}(x, y)=L_{Y U} U_{0}(x)+L_{Y V} V_{0}(x)+L_{Y Y} Y_{0}(x)+L_{Y X} X_{0}(x), \\
& X(x, y)=\tau_{x y}(x, y)=L_{X U} U_{0}(x)+L_{X V} V_{0}(x)+L_{X Y} Y_{0}(x)+L_{X X} X_{0}(x), \\
& \sigma_{x}(x, y)=A_{U} U_{0}(x)+A_{V} V_{0}(x)+A_{Y} Y_{0}(x)+A_{X} X_{0}(x),
\end{aligned}
$$

here, $U_{0}(x)=G u(x, 0), V_{0}(x)=G v(x, 0), Y_{0}(x)=\sigma_{y}(x, 0), X_{0}(x)=\tau_{x y}(x, 0)$ are the initial functions.

Differential operators can be expressed in the form of infinite series or by symbolic formulas in the case of plane strain [14]

$$
\begin{aligned}
& L_{U U}=\cos (\alpha y)-\frac{\alpha y}{2(1-v)} \sin (\alpha y), \\
& L_{U V}=-\frac{(1-2 v)}{2(1-v)} \sin (\propto y)-\frac{\propto y}{2(1-v)} \cos (\propto y), \\
& L_{U Y}=-\frac{y}{4(1-v)} \sin (\propto y), \\
& L_{U X}=\frac{1}{\propto} \sin (\propto y)-\frac{\sin (\propto y)}{4 \propto(1-v)}-\frac{\propto y \cos (\propto y)}{4 \propto(1-v)}, \\
& L_{V U}=L_{U V}, \\
& L_{V V}=\frac{\propto y}{2(1-v)} \sin (\propto y)+\cos (\propto y), \\
& L_{V Y}=\frac{(3-4 v) \sin (\propto y)}{4(1-v) \propto}-\frac{y}{4(1-v)} \cos (\propto y), \\
& L_{V X}=L_{U Y}, \\
& L_{Y U}=\frac{\propto^{2} y}{1-v} \sin (\propto y), \\
& L_{Y V}=\frac{\propto}{1-v}[\sin (\propto y)-\propto y \cos (\propto y)], \\
& L_{Y Y}=L_{V V}, \\
& L_{Y X}=L_{U V},
\end{aligned}
$$




$$
\begin{aligned}
& L_{X U}=-\frac{\propto}{1-v}[\sin (\propto y)+\propto y \cos (\propto y)], \\
& L_{X V}=L_{Y U}, \\
& L_{X Y}=L_{V U}, \\
& L_{X X}=L_{U U}, \\
& A_{U}=\frac{2 \propto}{1-v} \cos (\propto y)-\frac{y \propto 2}{1-v} \sin (\propto y), \\
& A_{V}=-\frac{\propto}{1-v}[\sin (\propto y)+\propto y \cos (\propto y)], \\
& A_{Y}=\frac{v}{1-v} \cos (\propto y)-\frac{y \propto}{2(1-v)} \sin (\propto y), \\
& A_{X}=\frac{y \propto}{2(1-v)} \cos (\propto y)+\frac{3-2 v}{2(1-v)} \sin (\propto y) .
\end{aligned}
$$

Here, $\quad \propto=\frac{\partial}{\partial x}$.

\section{Results and discussion}

We will get the symbolic solution satisfying the following boundary conditions

$$
\begin{aligned}
& V(x, 0)=V_{0}(x)=0, \\
& X(x, 0)=X_{0}(x)=0, \\
& V(x, h)=V_{h}(x), \\
& X(x, h)=X_{h}(x)=0 .
\end{aligned}
$$

Taking into account the symbolic Vlasov's solution satisfying the boundary conditions (3.1)-(3.4), we get

$$
\begin{aligned}
& 0=V_{0}(x), \\
& 0=X_{0}(x),
\end{aligned}
$$




$$
\begin{aligned}
& V_{h}(x)=\frac{1}{4(1-v) \propto}\left\{\left[2 h \propto^{2} \sin (h \propto)+4(1-v) \propto \cos (h \propto)\right] V_{0}(x)+\right. \\
& +h \propto \sin (h \propto) X_{0}(x)+((3-4 v) \sin (h \propto)-h \propto \cos (h \propto)) Y_{0}(x)+ \\
& \left.+\left[2(1-2 v) \propto \sin (h \propto)-2 h \propto^{2} \cos (h \propto)\right] U_{0}(x)\right\}, \\
& 0=\frac{1}{2(1-v)}\left\{2 h \propto^{2} \sin (h \propto) V_{0}(x)+[2(1-v) \cos (h \propto)-h \propto \sin (h \propto)] X_{0}(x)+\right. \\
& \left.+((1-2 v) \sin (h \propto)-h \propto \cos (h \propto)) Y_{0}(x)-\left[2 \propto \sin (h \propto)+2 h \propto^{2} \cos (h \propto)\right] U_{0}(x)\right\} .
\end{aligned}
$$

The differential operators will be treated in the same way as the trigonometric functions. So, we get a system of four Eqs (3.5)-(3.8) whose solution depending on the four initial functions is given by

$$
\begin{aligned}
& U_{0}(x)=\frac{1}{2(1-v)} \frac{(1-2 v) \sin (h \propto)-h \propto \cos (h \propto)}{\sin ^{2}(h \propto)} V_{0}(x), \\
& V_{0}(x)=0 \\
& Y_{0}(x)=\frac{1}{1-v} \frac{h \propto^{2} \cos (h \propto)+\propto \sin (h \propto)}{\sin ^{2}(h \propto)} V_{h}(x), \\
& X_{0}(x)=0 .
\end{aligned}
$$

Using expressions (3.9)-(3.12) in the symbolic solution of the initial functions, we get the solution for the band satisfying the boundary conditions (3.1)-(3.4)

$$
\begin{aligned}
& U(x, y)=\frac{1}{2(1-v)}\left[y \frac{\propto \sin (y \propto)}{\sin (h \propto)}-(1-2 v) \frac{\cos (y \propto)}{\sin (h \propto)}+h \frac{\propto \cos (h \propto) \cos (y \propto)}{(\sin (h \propto))^{2}}\right] V_{h}(x), \\
& V(x, y)=\frac{1}{2(1-v)}\left[2(1-v) \frac{\sin (y \propto)}{\sin (h \propto)}-y \frac{\propto \cos (y \propto)}{\sin (h \propto)}+h \frac{\propto \cos (h \propto) \sin (y \propto)}{(\sin (h \propto))^{2}}\right] V_{h}(x), \\
& Y(x, y)=\frac{1}{1-v}\left[\frac{\propto \cos (y \propto)}{\sin (h \propto)}+y \frac{\propto^{2} \sin (y \propto)}{\sin (h \propto)}+h \frac{\propto^{2} \cos (h \propto) \cos (y \propto)}{(\sin (h \propto))^{2}}\right] V_{h}(x), \\
& X(x, y)=\frac{1}{1-v}\left[h \frac{\propto^{2} \cos (h \propto) \sin (y \propto)}{(\sin (h \propto))^{2}}-y \frac{\propto^{2} \cos (\mathrm{y} \propto)}{\sin (h \propto)}\right] V_{h}(x),
\end{aligned}
$$




$$
\sigma_{x}(x, y)=\frac{1}{1-v}\left[\frac{\propto \cos (y \propto)}{\sin (h \propto)}-y \frac{\propto^{2} \sin (y \propto)}{\sin (h \propto)}-h \frac{\propto^{2} \cos (h \propto) \cos (y \propto)}{(\sin (h \propto))^{2}}\right] V_{h}(x) .
$$

The obtained relationships satisfy the equilibrium equations, strain and stress equations, as well as the biharmonic equation.

\subsection{Analysis of harmonic functions}

We can point out that each term of the symbolic solution (3.13)-(3.17) is a harmonic function. This allows us to substitute the previous function by the exact solution of the harmonic equation.

The symbolic solution (3.13)-(3.17) obtained for a band has the following form [15]

$$
\begin{aligned}
& \frac{\sin (y \propto)}{\sin (h \propto)} V_{h}(x), \\
& \frac{\cos (y \propto)}{\sin (h \propto)} V_{h}(x)=\frac{1}{\propto} \frac{\partial}{\partial y}\left[\frac{\sin (y \propto)}{\sin (h \propto)} V_{h}(x)\right]=\int \frac{\partial}{\partial y}\left[\frac{\sin (y \propto)}{\sin (h \propto)} V_{h}(x)\right] d x, \\
& \frac{\propto \cos (y \propto)}{\sin (h \propto)} V_{h}(x)=\propto\left[\frac{\sin (y \propto)}{\sin (h \propto)} V_{h}(x)\right]=\frac{\partial}{\partial x}\left[\frac{\sin (y \propto)}{\sin (h \propto)} V_{h}(x)\right], \\
& \frac{\propto \cos (y \propto)}{\sin (h \propto)} V_{h}(x)=\frac{\partial}{\partial y}\left[\frac{\sin (y \propto)}{\sin (h \propto)} V_{h}(x)\right], \\
& \frac{\propto^{2} \sin (y \propto)}{\sin (h \propto)} V_{h}(x)=\propto^{2}\left[\frac{\sin (y \propto)}{\sin (h \propto)} V_{h}(x)\right]=\frac{\partial^{2}}{\partial x^{2}}\left[\frac{\sin (y \propto)}{\sin (h \propto)} V_{h}(x)\right], \\
& \frac{\propto^{2} \cos (y \propto)}{\sin (h \propto)} V_{h}(x)=\propto \frac{\partial}{\partial y}\left[\frac{\sin (y \propto)}{\sin (h \propto)} V_{h}(x)\right]=\frac{\partial^{2}}{\partial x \partial y}\left[\frac{\sin (y \propto)}{\sin (h \propto)} V_{h}(x)\right], \\
& \frac{\propto \cos (h<) \cos (y \propto)}{(\sin (h \propto))^{2}} V_{h}(x)=-\frac{\partial}{\partial h}\left[\frac{\cos (y \propto)}{\sin (h \propto)} V_{h}(x)\right]=-\int \frac{\partial^{2}}{\partial h \partial y}\left[\frac{\sin (y \propto)}{\sin (h \propto)} V_{h}(x)\right] d x, \\
& \frac{\propto^{2} \cos (h \propto) \cos (y \propto)}{(\sin (h \propto))^{2}} V_{h}(x)=-\propto \frac{\partial}{\partial h}\left[\frac{\cos (h \propto)}{\sin (h \propto)} V_{h}(x)\right]=-\frac{\partial^{2}}{\partial h \partial y}\left[\frac{\sin (y \propto)}{\sin (h \propto)} V_{h}(x)\right], \\
& \frac{\propto \cos (h \propto) \sin (y \propto)}{(\sin (h \propto))^{2}} V_{h}(x)=-\frac{\partial}{\partial h}\left[\frac{\sin (y \propto)}{\sin (h \propto)}\right] V_{h}(x),
\end{aligned}
$$




$$
\frac{\propto^{2} \cos (h \propto) \sin (y \propto)}{(\sin (h \propto))^{2}} V_{h}(x)=-\propto \frac{\partial}{\partial h}\left[\frac{\sin (y \propto)}{\sin (h \propto)} V_{h}(x)=-\frac{\partial^{2}}{\partial h \partial y}\left[\frac{\sin (y \propto)}{\sin (h \propto)} V_{h}(x)\right]\right] .
$$

Moreover, it is known that if a harmonic function $f$ is defined in a domain:

$0 \leq x \leq l, 0 \leq y \leq h$, and satisfies the following boundary conditions

$$
\begin{aligned}
& f(0, y)=f_{1}(y), \\
& f(l, y)=f_{2}(y), \\
& f(x, 0)=f_{3}(x), \\
& f(x, h)=f_{4}(x),
\end{aligned}
$$

its expression has the following form [16]

$$
\begin{aligned}
& f(x, y)=\sum_{n=1}^{\infty} A_{n} \sinh \left[\frac{n \pi(l-x)}{h}\right] \sin \left(\frac{n \pi y}{h}\right)+\sum_{n=1}^{\infty} B_{n} \sinh \left(\frac{n \pi x}{h}\right) \sin \left(\frac{n \pi y}{h}\right)+ \\
& +\sum_{n=1}^{\infty} C_{n} \sin \left(\frac{n \pi x}{l}\right) \sinh \left[\frac{n \pi(h-y)}{l}\right]+\sum_{n=1}^{\infty} D_{n} \sin \left(\frac{n \pi x}{l}\right) \sinh \left(\frac{n \pi y}{l}\right)
\end{aligned}
$$

here the coefficients $A_{n}, B_{n}, C_{n}, D_{n}$ are determined as follows [16]

$$
\begin{aligned}
& A_{n}=\frac{2}{\lambda_{n}} \int_{0}^{h} f_{1}(\xi) \sin \left(\frac{n \pi \xi}{h}\right) d \xi, \\
& B_{n}=\frac{2}{\lambda_{n}} \int_{0}^{h} f_{2}(\xi) \sin \left(\frac{n \pi \xi}{h}\right) d \xi, \\
& C_{n}=\frac{2}{\mu_{n}} \int_{0}^{l} f_{3}(\xi) \sin \left(\frac{n \pi \xi}{l}\right) d \xi, \\
& D_{n}=\frac{2}{\mu_{n}} \int_{O}^{l} f_{4}(\xi) \sin \left(\frac{n \pi \xi}{l}\right) d \xi,
\end{aligned}
$$

Here

$$
\lambda_{n}=h \sinh \left(\frac{n \pi l}{h}\right), \quad \mu_{n}=l \sinh \left(\frac{n \pi h}{l}\right)
$$

Now consider the harmonic function (3.18) 


$$
f(x, y)=\frac{\sin (y \alpha)}{\sin (h \alpha)} V_{h}(x) .
$$

We get

$$
\begin{aligned}
& f_{l}(y)=f(0, y)=\frac{\sin (y \alpha)}{\sin (h \alpha)} V_{h}(0)=\left(\frac{y}{h}+\frac{y h^{2}-H^{3}}{6 h} \alpha^{2}+O\left(\alpha^{4}\right)\right) V_{h}(0)= \\
& =\left(\frac{y}{h}+\frac{y h^{2}-y^{3}}{6 h} \frac{\partial^{2}}{\partial x^{2}}+\ldots\right) V_{h}(0)=\frac{y}{h} V_{h}(0), \\
& f_{2}(y)=f(l, y)=\frac{\sin (\propto y)}{\sin (h y)} V_{h}(l)=\left(\frac{y}{h}+\frac{y h^{2}-y^{3}}{6 h} \propto^{2}+O\left(\propto^{4}\right)\right) V_{h}(l)= \\
& =\left(\frac{y}{h}+\frac{y h^{2}-y^{3}}{6 h} \frac{\partial^{2}}{\partial x^{2}}+\ldots\right) V_{h}(l)=\frac{y}{h} V_{h}(l), \\
& f_{3}(x)=f(x, 0)=\frac{\sin (0 \propto)}{\sin (h \propto)} V_{h}(x)=0, \\
& f_{4}(x)=f(x, h)=\frac{\sin (h \propto)}{\sin (h \propto)} V_{h}(x)=V_{h}(x) .
\end{aligned}
$$

Finally, the expression of the solution has the form

$$
\begin{aligned}
& f(x, y)=\sum_{n=1}^{\infty} A_{n} \sinh \left[\frac{n \pi(l-x)}{h}\right] \sin \left(\frac{n \pi y}{h}\right)+\sum_{n=1}^{\infty} B_{n} \sinh \left(\frac{n \pi x}{h}\right) \sin \left(\frac{n \pi y}{h}\right)+ \\
& +\sum_{n=1}^{\infty} C_{n} \sin \left(\frac{n \pi x}{l}\right) \sinh \left[\frac{n \pi(h-y)}{l}\right]+\sum_{n=1}^{\infty} D_{n} \sin \left(\frac{n \pi x}{l}\right) \sinh \left(\frac{n \pi y}{l}\right),
\end{aligned}
$$

here

$$
\begin{aligned}
& A_{n}=\frac{2 V_{h}(0)}{\lambda_{n}} \int_{0}^{h} \frac{\xi}{h} \sin \left(\frac{n \pi \xi}{h}\right) d \xi, \\
& B_{n}=\frac{2 V_{h}(l)}{\lambda_{n}} \int_{0}^{h} \frac{\xi}{h} \sin \left(\frac{n \pi \xi}{h}\right) d \xi \\
& C_{n}=0
\end{aligned}
$$




$$
D_{n}=\frac{2}{\mu_{n}} \int_{0}^{h} V_{h}(\xi) \sin \left(\frac{n \pi \xi}{l}\right) d \xi
$$

\subsection{Implementation on a rectangular plate under local surface loading}

Our goal is to study the plate under a local surface load on the plane $y=h$. We will consider that normal displacements under load are a known variable function of $x$. On the plane $y=0$, displacements vanish. On the other hand, on the planes $y=0$ and $y=h$ the shear stresses are zero. The boundary conditions of the problem are as follows [14]

$$
\begin{aligned}
& v(0, y)=\frac{1}{G} V(0, y)=v(l, y)=\frac{1}{G} V(l, y)=0, \\
& \sigma_{y}(0, y)=Y(0, y)=\sigma_{y}(l, y)=Y(l, y)=0, \\
& \sigma_{x}(0, y)=\sigma_{x}(l, y)=0, \\
& v(x, h)=\frac{1}{G} V_{h}(x), \\
& v(x, 0)=\frac{1}{G} V_{0}(x)=0, \\
& \tau_{x y}(x, h)=X_{h}(x)=\tau_{x y}(x, 0)=X_{0}(x)=0 .
\end{aligned}
$$

When condition (3.46) is satisfied for all $y$, we can write the following equality

$$
\left.V(0, y)\right|_{y=h}=0 .
$$

Also we have

$$
\begin{aligned}
& \left.V(0, y)\right|_{y=h}=V_{0}(0, h)=V_{h}(0)=0, \\
& \left.V(l, y)\right|_{y=h}=V(l, h)=V_{h}(l)=0 .
\end{aligned}
$$

Substituting Eqs (3.52) and (3.53) in expressions (3.42)- (3.45), one obtains

$$
\begin{aligned}
& A_{n}=B_{n}=C_{n}=0 \\
& D_{n}=\frac{2}{l \sinh \left(\frac{n \pi h}{l}\right)} \int_{0}^{l} V_{h}(\xi) \sin \left(\frac{n \pi \xi}{l}\right) d \xi .
\end{aligned}
$$

Finally, we get 


$$
\frac{\sin (y \alpha)}{\sin (h \alpha)} V_{h}(x)=\sum_{n=1}^{\infty} \frac{\sin \left(\frac{n \pi x}{l}\right) \sinh \left(\frac{n \pi y}{l}\right)}{\sinh \left(\frac{n \pi h}{l}\right)} \frac{2}{l} \int_{0}^{l} V_{h}(\xi) \sin \left(\frac{n \pi \xi}{l}\right) d \xi
$$

Using expressions (3.19)-(3.27), we find solutions for other harmonic functions

$$
\begin{aligned}
& \frac{\cos (y \propto)}{\sin (h \propto)} V_{h}(x)=-\sum_{n=1}^{\infty} \frac{\cosh \left(\frac{n \pi y}{l}\right) \cos \left(\frac{n \pi x}{l}\right)}{\sinh \left(\frac{n \pi h}{l}\right)} \frac{2}{l} \int_{0}^{l} V_{h}(\xi) \sin \left(\frac{n \pi \xi}{l}\right) d \xi, \\
& \frac{\alpha \sin (y \alpha)}{\sin (h \alpha)} V_{h}(x)=\sum_{n=1}^{\infty} \frac{n \pi}{l} \frac{\cos \left(\frac{n \pi x}{l}\right) \sinh \left(\frac{n \pi y}{l}\right)}{\sinh \left(\frac{n \pi h}{l}\right)} \frac{2}{l} \int_{0}^{l} V_{h}(\xi) \sin \left(\frac{n \pi \xi}{l}\right) d \xi, \\
& \frac{\alpha \cos (y \alpha)}{\sin (h \alpha)} V_{h}(x)=\sum_{n=1}^{\infty} \frac{n \pi}{l} \frac{\sin \left(\frac{n \pi x}{l}\right) \cosh \left(\frac{n \pi y}{l}\right)}{\sinh \left(\frac{n \pi h}{l}\right)} \frac{2}{l} \int_{0}^{l} V_{h}(\xi) \sin \left(\frac{n \pi \xi}{l}\right) d \xi, \\
& \frac{\alpha^{2} \sin (y \alpha)}{\sin (h \alpha)} V_{h}(x)=-\sum_{n=1}^{\infty} \frac{n^{2} \pi^{2}}{l^{2}} \frac{\sin \left(\frac{n \pi x}{l}\right) \sinh \left(\frac{n \pi y}{l}\right)}{\sinh \left(\frac{n \pi h}{l}\right)} \frac{2}{l} \int_{0}^{l} V_{h}(\xi) \sin \left(\frac{n \pi \xi}{l}\right) d \xi, \\
& \frac{\alpha^{2} \cos (y \alpha)}{\sin (h \alpha)} V_{h}(x)=\sum_{n=1}^{\infty} \frac{n^{2} \pi^{2}}{l^{2}} \frac{\cos \left(\frac{n \pi x}{l}\right) \cosh \left(\frac{n \pi y}{l}\right)}{\sinh \left(\frac{n \pi h}{l}\right)} \frac{2}{l} \int_{0}^{l} V_{h}(\xi) \sin \left(\frac{n \pi \xi}{l}\right) d \xi, \\
& \frac{\alpha \cos (h \alpha) \cos (y \alpha)}{(\sin (h \alpha))^{2}} V_{h}(x)= \\
& =-\sum_{n=1}^{\infty} \frac{n \pi}{l} \frac{\cosh \left(\frac{n \pi y}{l}\right) \cos \left(\frac{n \pi x}{l}\right) \cosh \left(\frac{n \pi h}{l}\right)}{l} \frac{2}{l} \int_{0}^{l} V_{h}(\xi) \sin \left(\frac{n \pi \xi}{l}\right) d \xi,
\end{aligned}
$$




$$
\begin{aligned}
& \frac{\alpha^{2} \cos (h \alpha) \cos (y \alpha)}{(\sin (h \alpha))^{2}} V_{h}(x)= \\
& =\sum_{n=1}^{\infty} \frac{n^{2} \pi^{2}}{l^{2}} \frac{\sin \left(\frac{n \pi x}{l}\right) \cosh \left(\frac{n \pi y}{l}\right) \cosh \left(\frac{n \pi h}{l}\right)}{2} \frac{2}{l} \int_{0}^{l} V_{h}(\xi) \sin \left(\frac{n \pi \xi}{l}\right) d \xi \\
& \frac{\alpha \cos (h \alpha) \sin (h \alpha)}{(\sin (h \alpha))^{2}} V_{h}(x)= \\
& =\sum_{n=1}^{\infty} \frac{n \pi}{l} \frac{\sinh \left(\frac{n \pi y}{l}\right) \sin \left(\frac{n \pi x}{l}\right) \cosh \left(\frac{n \pi h}{l}\right)}{2} \frac{2}{l} \int_{0}^{l} V_{h}(\xi) \sin \left(\frac{n \pi \xi}{l}\right) d \xi \\
& \frac{\alpha^{2} \cos (h \alpha) \sin (y \alpha)}{(\sin (h \alpha))^{2}} V_{h}(x)= \\
& =\sum_{n=1}^{\infty} \frac{n^{2} \pi^{2}}{l^{2}} \frac{\cos \left(\frac{n \pi x}{l}\right) \sinh \left(\frac{n \pi y}{l}\right) \cosh \left(\frac{n \pi h}{l}\right)}{l} \frac{2}{l} \int_{0}^{l} V_{h}(\xi) \sin \left(\frac{n \pi \xi}{l}\right) d \xi . \\
& \left(\sinh \left(\frac{n \pi h}{l}\right)\right)^{2}
\end{aligned}
$$

Substituting expressions (3.56)-(3.65) into (3.13)-(3.17), we find expressions for stresses and displacements [14]

$$
\begin{aligned}
& U(x, y)=\sum_{n=1}^{\infty} \frac{\delta_{n} \cos \left(\beta_{n} \xi\right)}{2(1-v) \sinh ^{2}\left(\beta_{n}\right)}\left[\left((1-2 v) \sinh \left(\beta_{n}\right)+\right.\right. \\
& \left.\left.-\beta_{n} \cosh \left(\beta_{n}\right)\right) \cosh \left(\beta_{n} \eta\right)+\beta_{n} \sinh \left(\beta_{n}\right) \sinh \left(\beta_{n} \eta\right)\right] \\
& V(x, y)=\sum_{n=1}^{\infty} \frac{\delta_{n} \sin \left(\beta_{n} \xi\right)}{2(1-v) \sinh ^{2}\left(\beta_{n}\right)}\left[\left(2(1-v) \sinh \left(\beta_{n}\right)+\right.\right. \\
& \left.+\beta_{n} \cosh \left(\beta_{n}\right) \operatorname{sh}\left(\beta_{n} \eta\right)-\beta_{n} \sinh \left(\beta_{n}\right) \eta \cosh \left(\beta_{n} \eta\right)\right] \\
& Y(x, y)=\sum_{n=1}^{\infty} \frac{\delta_{n} \beta_{n} \sin \left(\beta_{n} \xi\right)}{h(1-v) \sinh ^{2}\left(\beta_{n}\right)}\left[\left(\sinh \left(\beta_{n}\right)+\right.\right. \\
& \left.+\beta_{n} \cosh \left(\beta_{n}\right) \cosh \left(\beta_{n} \eta\right)-\beta_{n} \sinh \left(\beta_{n}\right) \eta \cosh \left(\beta_{n} \eta\right)\right] \\
& X(x, y)=\sum_{n=1}^{\infty} \frac{\delta_{n} \beta_{n}^{2} \cos \left(\beta_{n} \xi\right)}{h(1-v) \sinh ^{2}\left(\beta_{n}\right)}\left[\cosh \left(\beta_{n}\right) \sinh \left(\beta_{n} \eta\right)-\sinh \left(\beta_{n}\right) \eta \cosh \left(\beta_{n} \eta\right)\right]
\end{aligned}
$$


here,

$$
\eta=\frac{y}{h}, \quad \xi=\frac{x}{h}, \quad \beta_{n}=\frac{n \pi h}{l}, \quad \delta_{n}=\frac{2}{l} \int_{0}^{l} V_{h}(\xi) \sin \left(\frac{n \pi \xi}{l}\right) d \xi
$$

The use of the method of initial functions and the replacement of harmonic functions with corresponding solutions allows us to obtain expressions for stresses and displacements. The efficiency of this algorithm is shown in the problem of a plate under local surface loading. Thus, having replaced the harmonic functions presented in symbolic form with the corresponding solutions of the harmonic equation for a rectangle, we get the solution to the problem in trigonometric form.

\section{Conclusion}

From this work we can draw the following conclusions:

1. An analysis approach to the harmonic problem of a plate in plane strain using the symbolic method of initial functions and the solution of the harmonic equation for a rectangle is proposed;

2. A technique to introduce Vlasov's symbolic solution for a band into the solution of the harmonic equation for a rectangle in order to get the exact solution of the problem is proposed;

3. An algorithm is developed for the determination of expressions of displacements and stresses in trigonometric series for a plate subjected to a local surface loading.

\section{Nomenclature}

$$
\begin{aligned}
L & - \text { differential operators matrix } \\
L_{U U} ; L_{U V} ; \ldots ; L_{X Y} & - \text { differential operators related to the initial functions } \\
U_{0}(0), V_{0}(0), & \text {-initial functions } \\
Y_{0}(0), X_{0}(0) & \\
W & - \text { displacement vector } \\
\sigma & - \text { stress vector }
\end{aligned}
$$

\section{References}

[1] Soldatenkov I.A. (2016): The three-dimensional problem on the mutual wear of a thin elastic layer and punch sliding on it. - Journal of Applied Mathematics and Mechanics, vol.80, No.1, pp.117-137.

[2] Saidi A.R., Jomehzadeh E. and Atashipour S.R. (2009): Exact analytical solution for bending analysis of functionally graded annular sector plates. - International Journal of Engineering, Translation B: Applications, vol.22, No.3, pp.307-316.

[3] Jomehzadeh E., Saidi A.R. and Atashipour S.R. (2009): Analytical approach for stress analysis of functionally graded annular sector plates. - Materials and Design, vol.30, No.9, pp.3679-3685.

[4] Yang B., Ding H.J. and Chen W.Q. (2009): Elasticity solutions for a uniformly loaded rectangular plate of functionally graded materials with opposite edges simply supported. - Acta Mechanica, vol.207, No.3, pp.245-258.

[5] Maliev A.S. (1951): Some problems of the bending theory of rectangular plates. - Abstract of doctor of science thesis -Leningrad

[6] Vlasov V.Z. (1955): The method of initial functions in problems of theory of elasticity. - Bulletin of the Academy of Science of the USSR, vol.7, pp.49-69.

[7] Kellog O.G. (1951): On the derivatives of harmonic functions on the boundary. - Transaction of American Mathematical Society, vol.39, pp.406-510. 
[8] Faraji S., Lowell and Archer R.R. (1989): Method of initial functions for thick transversely isotropic shells. Archive of Applied Mechanics, vol.60, No.1, pp.1-9.

[9] Sargand S.M., Chen N.N. and Das Y.C. (1992): Method of initial functions for axially symmetric elastic bodies. International Journal of Solids and Structures, vol.29, No.6, pp.711-719.

[10] Chandrashekhara K. and Nanjunda Rao K.S. (1998): Method of initial functions for the analysis of laminated circular cylindrical shell under axisymmetric load. - Mechanics of Composite Materials and Structures, vol.5, No.2, pp.187-201.

[11] Patel R., Dubey S.K. and Pathak K.K. (2012): Analusis of Composite beams using method of initial functions. International Journal of Advanced Structures and Geotechnical Engineering, vol.1, No.02,

[12] Patel R., Dubey S.K. and Pathak K.K. (2013): Analysis of flexural members using an alternative approach. Research Journal of Engineering Science, vol.2,N.4, pp.40-42.

[13] Matrosov V.A. (2012): Convergence of power series in the method of initial functions. - Bulletin of S.-Petersburg University, Applied Mathematics Series, vol.10, No.1, pp.41-51.

[14] Vlasov V.Z. and Leontiev U.N. (1966): Beams, plates and shells on elastic foundation. - Jerusalem: Israel Program for Scientific Translations.

[15] Zeldovich Y.B. (1977): Elements of Applied Mathematics. - Amazon.

[16] Polianin A.D. (2001): Spravochnik po lineinym uravneniiam matematicheskoy fiziki. - M.: Fizmatlit. (In Russian).

Received: September 9, 2016

Revised: March 13, 2017 